\title{
Micronutrientes metálicos nos moluscos bivalves Crassostrea rhizophorae e Mytella guyanensis
}

\author{
Glaucia Batista Maciel dos Santos®, Guisla Boehs*® \\ a Programa de Pós-Graduação em Ciência Animal, Universidade Estadual de Santa Cruz, Ilhéus, 45662-900, Bahia, Brasil. \\ *guislaboehs@hotmail.com
}

Received: 18 maio 2021 / Accepted: 5 agosto 2021 / Published online: 30 setembro 2021

\begin{abstract}
Resumo
Os micronutrientes, micro ou oligoelementos, incluem vitaminas e alguns metais, sendo que uma parcela significativa da população mundial apresenta deficiência nutricional desses elementos. Este estudo avaliou os teores de micronutrientes metálicos em Crassostrea rhizophorae (ostra) e Mytella guyanensis (sururu) coletados no sul da Bahia, Brasil, num trecho de $280 \mathrm{~km}$ de extensão. Os teores dos metais ( $\mathrm{Cu}, \mathrm{Fe}, \mathrm{Mn}$ e $\mathrm{Zn})$ da parte edível das espécies foram avaliados em espectrometria de emissão óptica de plasma indutivamente acoplado (ICP OES). As concentrações médias encontradas para C. rhizophorae e M. guyanensis, respectivamente, foram: $\mathrm{Cu}(2,09 \pm 0,90$ e 2,31 $\pm 0,63)$; $\mathrm{Fe}(32,03 \pm 4,18$ e 213,36 $\pm 51,67) ; \mathrm{Mn}(1,75 \pm 0,44 \mathrm{e}$ $13,61 \pm 2,83)$ e Zn $(129,54 \pm 25,88$ e 12,13 $\pm 1,13)$, em mg kg-1. Ambos os bivalves foram relevantes na concentração dos micronutrientes investigados, com destaque para os valores elevados de $\mathrm{Zn}$ em C. rhizophorae e de Fe em M. guyanensis.
\end{abstract}

Palavras-chave: Cobre, elementos essenciais, ferro, manganês, zinco.

\section{Metallic micronutrients in bivalve molluscs Crassostrea rhizophorae and Mytella guyanensis}

\begin{abstract}
Micronutrients, micro or oligo elements, include vitamins and some metals, being that a significant portion of the world population is nutritionally deficient in these elements. This study evaluated the levels of metallic micronutrients in Crassostrea rhizophorae (oyster) and Mytella guyanensis (mangrove mussel) collected in southern Bahia, Brazil, in a stretch of $280 \mathrm{~km}$ long. The metal contents $(\mathrm{Cu}, \mathrm{Fe}, \mathrm{Mn}$ and $\mathrm{Zn})$ of the edible part of the species were evaluated with inductively coupled plasma optical emission spectrometry (ICP OES). Mean concentrations in $\mathrm{mg} \mathrm{kg}^{-1}$ found for C. rhizophorae and M. guyanensis, respectively, were: $\mathrm{Cu}(2.09 \pm 0.90$ and $2.31 \pm 0.63)$; Fe (32.03 \pm 4.18 and 213.36 \pm 51.67$)$; $\mathrm{Mn}(1.75 \pm 0.44$ and $13.61 \pm 2.83)$ and $\mathrm{Zn}(129.54 \pm 25.88$ and $12.13 \pm 1.13)$. Both bivalves were relevant in the concentration of micronutrients investigated, with emphasis on the high values of $\mathrm{Zn}$ in C. rhizophorae and $\mathrm{Fe}$ in M. guyanensis.
\end{abstract}

Keywords: Copper, essential elements, iron, manganese, zinc.

\section{Introdução}

Micronutrientes (oligoelementos, microelementos, microminerais ou elementos essenciais) são indispensáveis ao crescimento e ao funcionamento do organismo humano. A deficiência desses elementos constitui-se em um problema de Saúde Pública (Maggini, Pierre, \& Calder, 2018). Uma dieta pobre em micronutrientes pode resultar em comprometimento orgânico irreversível, mesmo mediante reposição (Gimenez, Oliveros, \& Gomez, 2011). Por exemplo, o cobre participa de atividades enzimáticas e é essencial ao desenvolvimento cerebral (Scheiber, Mercer, \& Dringen, 2014), o ferro é importante no combate à anemia e no fortalecimento do sistema imune (Maggini et al., 2018), o manganês atua como cofator em diversas reações enzimáticas (Goyer \& Clarkson, 2001) e o zinco atua em atividades proteicas, sendo que a deficiência desse nutriente implica em alopecia, perda de peso e diarreia (Muhamed \& Vadstrup, 2014).

Moluscos bivalves são apreciados mundialmente na dieta humana, principalmente por possuírem alto valor proteico e baixo teor lipídico (Prato, Biandolino, Parlapiano, Giandomenico, Denti, Calò, Spada, \& Di Leo, 2019) e serem, igualmente, ricos e diversos em microminerais (Tabakaeva, Tabakaev, \& Piekoszewski, 2018), podendo suprir necessidades nutricionais essenciais (RodríguezHernández, Zumbado, Henríquez-Hernández, Boada, \& Luzardo, 2019). A ingestão destes tem contribuído para a melhora de diversas enfermidades, como no tratamento de doenças inflamatórias, incluindo a artrite reumatoide (Lindqvist, Gjertsson, Eneljung, \& Winkvist, 2018) e no combate à desnutrição infantil (Correia, Veiga, Santos, 
Cavalcante, Sawaya, \& Florêncio, 2018). Há indicações, também, que a ingestão de bivalves contribui para a prevenção e recuperação de transtornos depressivos (LaChance \& Ramsey, 2018).

Diante de tantos benefícios, milhões de toneladas de bivalves são anualmente produzidas em diversas partes do mundo, principalmente via aquicultura (FAO, 2020). Dentre os bivalves comercialmente importantes da costa brasileira estão Crassostrea rhizophorae (Guilding, 1828) (Ostreidae), a ostrado-mangue e Mytella guyanensis (Lamarck, 1819) (Mytilidae), o sururu ou marisco-do-mangue (Boehs, Villalba, Ceuta, \& Luz, 2010). A primeira fixa-se sobre raízes aéreas de plantas de mangue, em especial sobre Rhizophorae mangle L. (Boehs, Luz \& Andrade, 2019) e M. guyanensis enterra-se no sedimento, próximo a raízes subterrâneas dessas plantas (Boehs et al., 2010).

Embora bivalves tenham sido contemplados em uma série de estudos realizados nos últimos anos no litoral sul do estado da Bahia nos aspectos da ecologia, reprodução, cultivo, patologias e mais recentemente, também quanto ao teor de elementos químicos, não há estudos com enfoque no valor nutricional desses moluscos em termos de micronutrientes metálicos. Assim como em outros locais da costa brasileira, o foco das pesquisas envolvendo metais está geralmente voltado para o aspecto da toxicidade. Nesse contexto, e partindo-se da premissa que moluscos bivalves são bons bioacumuladores de metais, este estudo avaliou os teores dos microelementos $\mathrm{Cu}$, $\mathrm{Fe}, \mathrm{Mn}$ e Zn em C. rhizophorae e M. guyanensis de estuários do sul da Bahia (Brasil).

\section{Material e Métodos}

\section{Coleta e preparo das amostras}

Os bivalves foram coletados em quatro locais (L) (manguezais) do litoral sul do estado da Bahia, nos municípios de Valença $\left(13^{\circ} 26^{\prime} \mathrm{S}\right.$; $\left.39^{\circ} 04^{\prime} \mathrm{W}\right)$ (L1), Taperoá $\left(13^{\circ} 28^{\prime} \mathrm{S}\right.$;

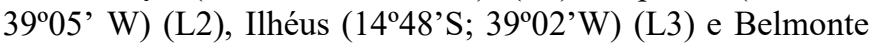
$\left(15^{\circ} 87^{\prime} \mathrm{S}\right.$; 38 $\left.86^{\prime} \mathrm{W}\right)(\mathrm{L} 4)$, num trecho de aproximadamente $280 \mathrm{~km}$ de extensão.

Os exemplares de $C$. rhizophorae foram coletados sobre raízes aéreas e caules do mangue vermelho $R$. mangle, ao passo que $M$. guyanensis foi coletada em substrato areno-lodoso próximo, ainda no manguezal. As coletas foram feitas manualmente, durante maré baixa, em dezembro de 2018, tendo sido previamente autorizadas pelo Instituto Chico Mendes da Biodiversidade e Conservação - ICMBio (concessão número 20912-6/2018).

As amostras foram armazenadas em sacos plásticos devidamente etiquetados e imediatamente processadas em laboratório. Após lavagem dos exemplares em água corrente, seu maior eixo (altura) foi medido com um paquímetro digital (Starfer, precisão de $0,01 \mathrm{~mm}$ ) e o peso individual, total (com concha) e somente do conteúdo intervalvar (carne), foram aferidos com balança de precisão (Marte, 0,001 g). O número de exemplares coletados por local e as informações biométricas das amostras estão expressas na tabela 1.

As vidrarias utilizada no experimento foram previamente lavadas com Ácido Nítrico $\left(\mathrm{HNO}_{3}\right)$ a $10 \%$ para descontaminação e posteriormente enxaguadas em água de osmose. Foi feito um pool de amostras, em triplicata, do conteúdo intervalvar de cada espécie, por ponto amostral, cujas amostras foram congeladas em freezer a $-80^{\circ} \mathrm{C}$ e em seguida liofilizadas (Labconco, modelo FreeZone 4.5) até peso constante.

Tabela 1. Informações sobre o local da coleta e tamanho amostral (n) e biometria (médias \pm desvio padrão) das espécies em estudo.

\begin{tabular}{ccccc}
\hline Local & $\mathrm{n}$ & $\begin{array}{c}\text { Altura da } \\
\text { concha }(\mathrm{cm})\end{array}$ & $\begin{array}{c}\text { Peso } \\
\text { total }(\mathrm{g})\end{array}$ & $\begin{array}{c}\text { Peso da } \\
\text { carne }(\mathrm{g})\end{array}$ \\
\hline \multicolumn{5}{c}{ Crassostrea rhizophora } \\
\hline L1 & 16 & $5,62 \pm 0,74$ & $18,59 \pm 5,95$ & $2,37 \pm 0,97$ \\
L2 & 15 & $6,53 \pm 1,04$ & $36,6 \pm 11,46$ & $5,01 \pm 1,70$ \\
L3 & 20 & $5,42 \pm 0,73$ & $18,86 \pm 5,31$ & $2,60 \pm 0,60$ \\
L4 & 12 & $9,28 \pm 1,78$ & $65,25 \pm 21,96$ & $6,66 \pm 1,89$ \\
\hline \multicolumn{5}{c}{ Mytella guyanensis } \\
\hline L1 & 30 & $4,60 \pm 0,48$ & $4,99 \pm 1,32$ & $1,60 \pm 0,42$ \\
L2 & 30 & $4,95 \pm 0,33$ & $5,77 \pm 1,19$ & $1,64 \pm 0,27$ \\
L3 & 30 & $4,97 \pm 0,45$ & $6,31 \pm 1,61$ & $1,80 \pm 0,46$ \\
L4 & 30 & $5,37 \pm 0,39$ & $7,98 \pm 1,49$ & $2,26 \pm 0,38$ \\
\hline Legenda : L1 - Valença, L2 - Taperoá, L3 - Ilhéus, L4 - Belmonte.
\end{tabular}

Após secas, as amostras foram trituradas e peneiradas em malha de nylon de 3,5 mm. Foram então pesados $250 \mathrm{mg}$ de cada pool de amostras, em triplicata, que foram transferidos para frascos de teflon (TFM), com adição posterior de $7 \mathrm{ml}$ de $\mathrm{HNO}_{3}$ a $65 \%$ e após 30 minutos de contato em temperatura ambiente $\left(25 \pm 1^{\circ} \mathrm{C}\right)$, foi adicionado $1 \mathrm{ml} \mathrm{de}$ peróxido de hidrogênio $\left(\mathrm{H}_{2} \mathrm{O}_{2}\right)$ a $30 \%$, após o que os frascos eram fechados e seguiam para o programa de digestão em forno microondas (modelo Mars Xpress-CEM). Foi utilizada temperatura de $90^{\circ} \mathrm{C}$ por 10 minutos em potência de 1.600 $\mathrm{W}$, seguido por um intervalo de 5 minutos, após o que a temperatura foi aumentada para $180^{\circ} \mathrm{C}$ por 15 minutos e nessa condição as amostras eram mantidas por mais 20 minutos após temperatura constante, com resfriamento posterior de 15 minutos. Após a digestão, as amostras foram transferidas para balões volumétricos, que foram preenchidos com água Milli-Q até a marca dos $25 \mathrm{ml}$. As soluções foram transferidas para tubos Falcon de $50 \mathrm{ml}$ e armazenadas em geladeira até a quantificação dos metais.

\section{Determinação analítica}

Para a determinação dos elementos $\mathrm{Cu}, \mathrm{Fe}, \mathrm{Mn}$ e $\mathrm{Zn}$, foi feito uso de um espectrômetro de emissão óptica com plasma indutivamente acoplado (ICP OES), modelo 710-ES (Varian Mulgrave, Austrália), equipado com um nebulizador concêntrico Meinhard® (Santa Clara, EUA), acoplado a uma câmara de nebulização ciclônica - single pass (Varian), tocha de quartzo (Varian) e detector de estado sólido com arranjo de diodos CCD. Foi utilizado Argônio 99,998\% (White Martins/Praxair, Bahia, Brasil), como gás gerador do plasma e do sistema de nebulização. Os parâmetros instrumentais estão apresentados na tabela 2.

Soluções estoque de padrões monoelementares da Specsol (Quimlab Química e Metrologia, São José dos 
Campos, Brasil) (1.000 mg $\mathrm{L}^{-1}$ e $10.000 \mathrm{mg} \mathrm{L}^{-1}$ ) foram utilizadas para preparar as curvas de calibração a partir de diluições apropriadas. Solução padrão multielementar Highpurity Standards (Carolina do Sul, EUA) foi utilizada para a calibração do sistema óptico do ICP OES. Não foi necessário o uso de padrão interno. As curvas de calibração foram construídas em uma faixa linear de 0,02 - 2,00 mg L $\mathrm{m}^{-1}$ Os cálculos para a determinação do Limite de Detecção (LD) e do Limite de Quantificação (LQ) foram feitos da seguinte forma: $L D=3,3 \frac{s}{s} ; L Q=3,3 \frac{s}{s}$, onde $s$ é o desvio padrão do sinal do branco analítico e $S$ é o coeficiente angular da curva de calibração.

Tabela 2. Parâmetros instrumentais e operacionais utilizados no espectrômetro de emissão óptica com plasma indutivamente acoplado (ICP OES) na determinação analítica.

\begin{tabular}{lc}
\hline \multicolumn{1}{c}{ Parâmetros Instrumentais } & Condições Operacionais \\
\hline Potência de radiofrequência $(\mathrm{kW})$ & 1,4 \\
Vazão do gás do plasma $\left(\mathrm{L} \mathrm{min}^{-1}\right)$ & 15,0 \\
Vazão do gás auxiliar $\left(\mathrm{L} \mathrm{min}^{-1}\right)$ & 1,5 \\
Pressão de nebulização $(\mathrm{kPa})$ & 150 \\
Tempo de estabilização $(\mathrm{s})$ & 15,0 \\
Tempo de leitura das replicatas $(\mathrm{s})$ & 5 \\
Número de replicatas & 3 \\
Detector & $\mathrm{CCD}$ \\
Velocidade da bomba peristáltica & 1 \\
$(\mathrm{rpm})$ &
\end{tabular}
(rpm)

Linhas de emissão (nm)

$\mathrm{Cu}:$ 327.395; Fe: 259.940; Mn: 257.610; Zn: 213.857

\section{Tratamento dos dados}

Os teores dos microelementos foram comparados entre espécies e entre locais através de análises de variância (ANOVAs), seguidas, quando necessário, pelo teste de Tukey. O nível de confiança utilizado foi de $95 \%$ e as análises foram feitas no programa estatístico BioEstat 5.0.

Como parâmetro para os valores máximos de metais permitidos pela legislação brasileira em alimentos, foi utilizado o Decreto n. 55.871 da Agência Nacional de Vigilância Sanitária do Ministério da Saúde - ANVISA (1965).

\section{Resultados e Discussão}

O cobre apresentou concentrações médias iguais a 2,09 \pm $0,90 \mathrm{mg} \mathrm{kg}^{-1}$ (1,33 a 3,35 mg kg-1) em C. rhizophorae e 2,31 $\pm 0,63 \mathrm{mg} \mathrm{kg}^{-1}\left(1,71\right.$ a $\left.3,13 \mathrm{mg} \mathrm{kg}^{-1}\right)$ em M. guyanensis. Houve similaridade $(\mathrm{p}=0,5375)$ nos teores desse elemento entre as espécies e o L4 (Belmonte) teve destaque nesse elemento, nas duas espécies (Tabela 3).

Apesar de ser um elemento essencial, os níveis de cobre para o consumo humano são normatizados, sendo que as concentrações encontradas estiveram dentro dos limites (máximo de $30 \mathrm{mg} \mathrm{kg}^{-1}$ ) estabelecidos pela legislação brasileira (Brasil, 1965). Os valores encontrados $\left(2,09 \mathrm{mg} \mathrm{kg}^{-1} \mathrm{em} C\right.$. rhizophorae e $2,31 \mathrm{mg} \mathrm{kg}^{-1} \mathrm{em} M$. guyanensis) estiveram em faixas de concentração relativamente próximas às encontradas em Nodipecten nodosus (Linnaeus, 1758) (Pectinidae) coletada na Ilha Grande (Rio de Janeiro, Brasil) $\left(3,30 \mathrm{mg} \mathrm{kg}^{-1}\right)$ (Lino,
Galvão, Longo, Azevedo-Silva, Dorneles, Torres, \& Malm, 2016), em Perna perna (Linnaeus, 1758) (Mytilidae) coletada na Baía de Santos (São Paulo, Brasil) $(1,63$ mg kg1) (Campolim, Henriques, \& Barbieri, 2018), assim como em Anomalocardia brasiliana (Gmelin, 1791) (Veneridae) $(2,63$ $\mathrm{mg} \mathrm{kg}^{-1}$ ) e em Iphigenia brasiliana (Lamarck, 1818) (Donacidae) $\left(2,48 \mathrm{mg} \mathrm{kg}^{-1}\right)$, ambas coletadas na Baía de Todos os Santos (Bahia, Brasil) (Barbosa, Brito, Santos, Santos, Teixeira, Araujo, \& Korn, 2019), mas foram muito menores às concentrações observadas em Lucina (= Phacoides) pectinata (Gmelin, 1791) (Lucinidae) coletada em região próxima a porto e indústrias na Baía de Todos os Santos (121,30 $\mathrm{mg} \mathrm{kg}^{-1}$ ) (Barbosa et al., 2019). Igualmente, foram muito menores ao valor obtido por Lino et al. (2016) para $P$. perna coletada na Baía de Guanabara (Rio de Janeiro) (698 mg kg-1), cujo valor extrapolou, em muito, os limites estabelecidos pela ANVISA (Brasil, 1965).

Tabela 3. Médias e desvio padrão, em mg kg-1, em peso úmido, dos micronutrientes $\mathrm{Cu}, \mathrm{Fe}, \mathrm{Mn}$ e $\mathrm{Zn}$ em dois bivalves de manguezais (L) do sul da Bahia, Brasil.

\begin{tabular}{ccccc}
\hline L & $\mathrm{Cu}$ & $\mathrm{Fe}$ & $\mathrm{Mn}$ & $\mathrm{Zn}$ \\
\hline \multicolumn{5}{c}{ Crassostrea rhizophorae } \\
\hline L1 & $1,57 \pm 0,04^{\mathrm{c}}$ & $30,36 \pm 0,26^{\mathrm{c}}$ & $1,85 \pm 0,05^{\mathrm{b}}$ & $108,43 \pm 1,22^{\mathrm{c}}$ \\
L2 & $1,33 \pm 0,04^{\mathrm{d}}$ & $36,32 \pm 0,24^{\mathrm{a}}$ & $1,38 \pm 0,03^{\mathrm{c}}$ & $137,88 \pm 1,90^{\mathrm{b}}$ \\
L3 & $2,13 \pm 0,09^{\mathrm{b}}$ & $26,99 \pm 0,76^{\mathrm{d}}$ & $2,35 \pm 0,06^{\mathrm{a}}$ & $109,34 \pm 3,58^{\mathrm{c}}$ \\
L4 & $3,35 \pm 0,04^{\mathrm{a}}$ & $34,46 \pm 0,54^{\mathrm{b}}$ & $1,44 \pm 0,04^{\mathrm{c}}$ & $162,51 \pm 0,88^{\mathrm{a}}$ \\
LT & $2,09 \pm 0,90$ & $32,03 \pm 4,18$ & $1,75 \pm 0,44$ & $129,54 \pm 25,88$ \\
& $(\mathrm{p}<0,0001)$ & $(\mathrm{p}=0,0001)$ & $(\mathrm{p}<0,0001)$ & $(\mathrm{p}<0,0001)$ \\
\hline & & Mytella guyanensis & \\
\hline L1 & $1,71 \pm 0,07^{\mathrm{c}}$ & $181,62 \pm 13,30^{\mathrm{b}}$ & $11,12 \pm 0,08^{\mathrm{c}}$ & $10,59 \pm 0,26^{\mathrm{b}}$ \\
L2 & $1,92 \pm 0,05^{\mathrm{c}}$ & $186,60 \pm 2,58^{\mathrm{b}}$ & $12,30 \pm 0,12^{\mathrm{c}}$ & $12,02 \pm 0,06^{\mathrm{a}}$ \\
L3 & $2,51 \pm 0,19^{\mathrm{b}}$ & $290,45 \pm 12,18^{\mathrm{a}}$ & $13,41 \pm 1,09^{\mathrm{b}}$ & $13,14 \pm 0,98^{\mathrm{a}}$ \\
L4 & $3,13 \pm 0,12^{\mathrm{a}}$ & $194,79 \pm 10,54^{\mathrm{b}}$ & $17,63 \pm 0,82^{\mathrm{a}}$ & $12,79 \pm 0,13^{\mathrm{a}}$ \\
LT & $2,31 \pm 0,63$ & $213,36 \pm 51,67$ & $13,61 \pm 2,83$ & $12,13 \pm 1,13$ \\
& $(\mathrm{p}=0,0002)$ & $(\mathrm{p}=0,0002)$ & $(\mathrm{p}=0,0003)$ & $(\mathrm{p}=0,0027)$ \\
\hline
\end{tabular}

Legenda: L1 - Valença, L2 - Taperoá, L3 - Ilhéus, L4 - Belmonte, LT Todos os locais (media geral). Valores médios com letras diferentes, para o mesmo elemento e espécie, apresentaram diferenças significativas $(\mathrm{p}<0,05)$ no teste de Tukey. Entre parênteses, o p-valor entre tratamentos (locais).

Nos moluscos bivalves o cobre está associado com metalotioneínas, proteínas que exercem a função de controle e eliminação (detoxificação) desse metal (Cai \& Wang, 2019) e esta é também a razão pela qual esses animais conseguem concentrar altos níveis desse elemento em seus organismos. No caso de ostras, Weng, Jiang e Wang (2019) mostraram que nesses bivalves o cobre é um importante micronutriente na fase inicial da vida, atuando, por exemplo, de forma significativa no crescimento dos filamentos branquiais. No organismo humano, entre outros benefícios, esse micronutriente é importante ao bom funcionamento do sistema imune (Macêdo, Amorim, Silva, \& Castro, 2010).

O ferro apresentou concentrações médias iguais a 32,03 $\pm 4,18 \mathrm{mg} \mathrm{kg}^{-1}\left(26,99\right.$ a $\left.36,32 \mathrm{mg} \mathrm{kg}^{-1}\right)$ em C. rhizophorae e $213,36 \pm 51,67 \mathrm{mg} \mathrm{kg}^{-1}\left(181,62\right.$ a $\left.290,45 \mathrm{mg} \mathrm{kg}^{-1}\right)$ em $M$. 
guyanensis, com diferença significativa $(p<0,0001)$ entre espécies, sendo que a segunda apresentou concentrações seis vezes maiores à primeira, com destaque para os valores altos em M. guyanensis no L3 (Ilhéus; Tabela 3).

Lino et al. (2016) verificaram concentrações de ferro similares às de $M$. guyanensis deste estudo em outro mitilídeo (P. perna) (197 $\mathrm{mg} \mathrm{kg}^{-1}$ ) coletado na Baía de Guanabara (RJ) e valor similar $\left(179,97 \mathrm{mg} \mathrm{kg}^{-1}\right)$ foi igualmente encontrado em P. perna em outro estudo, na Baía de Santos (SP) (Campolim et al., 2018). Representantes dessa família de moluscos parecem ser bons bioacumuladores desse elemento, quando comparados, por exemplo, com bivalves como $C$. rhizophorae (presente estudo) e $N$. nodosus, que apresentou concentração de $40 \mathrm{mg} \mathrm{kg}^{-1}$, em amostras provenientes da Ilha Grande (RJ) (Lino et al., 2016).

Não existe um limite máximo de ferro em alimentos estabelecido na legislação ANVISA. No organismo humano, esse micronutriente atua na resposta imunitária (Maggini et al., 2018), sendo que a carência deste pode causar, além da anemia (principal manifestação dessa deficiência), retardo intelectual, assim como o aumento à suscetibilidade a infecções (Goyer \& Clarkson, 2001).

O manganês apresentou concentrações médias iguais a 1,75 $\pm 0,44 \mathrm{mg} \mathrm{kg}^{-1}\left(1,38\right.$ a 2,35 $\left.\mathrm{m} \mathrm{kg}^{-1}\right)$ em C. rhizophorae e 13,61 $\pm 2,83 \mathrm{mg} \mathrm{kg}^{-1}\left(11,12\right.$ a $\left.17,63 \mathrm{mg} \mathrm{kg}^{-1}\right)$ em M. guyanensis ( $p<0,0001$ entre espécies), sendo que a segunda espécie apresentou concentrações de cerca de oito vezes maiores do que a primeira, com destaque para o L4 (Belmonte), onde ocorreu o maior valor, em M. guyanensis (Tabela 3).

Em P. perna coletada na Baía de Guanabara (RJ), Lino et al. (2016) observaram $22 \mathrm{mg} \mathrm{kg}^{-1}$ de manganês e Campolim et al. (2018) verificaram $2,33 \mathrm{mg} \mathrm{kg}^{-1}$ na Baía de Santos (SP) nesse mesmo mitilídeo, mostrando valores muito variáveis na mesma espécie em diferentes locais. Já $N$. nodosus, coletada na Ilha Grande (RJ) (Lino et al., 2016), apresentou concentração muito mais alta em relação às três espécies citadas $\left(76 \mathrm{mg} \mathrm{kg}^{-}\right.$ ${ }^{1}$ ). O manganês é um micronutriente presente em todos os organismos vivos, nos quais participa de diversas atividades enzimáticas (Goyer \& Clarkson, 2001). Não há limite máximo para o consumo desse metal estabelecido na legislação brasileira (Brasil, 1965).

O zinco apresentou concentrações médias iguais a 129,54 $\pm 25,88 \mathrm{mg} \mathrm{kg}^{-1}\left(108,43\right.$ a $\left.162,51 \mathrm{mg} \mathrm{kg}^{-1}\right)$ em C. rhizophorae e $12,13 \pm 1,13 \mathrm{mg} \mathrm{kg}^{-1}\left(10,59\right.$ a $\left.13,14 \mathrm{mg} \mathrm{kg}^{-1}\right)$ em $M$. guyanensis $(\mathrm{p}<0,0001$ entre espécies), portanto, a primeira apresentou 10 vezes mais zinco do que a segunda espécie, com destaque para o L4 (Belmonte), onde ocorreu o maior valor, em C. rhizophorae (Tabela 3).

Os valores de $\mathrm{Zn}$ em $C$. rhizophorae foram superiores ao valor máximo estabelecido pela ANVISA em alimentos (Brasil, 1965) (50 mg kg-1, em peso úmido). Na comparação do alto valor de $\mathrm{Zn}$ dessa espécie em relação a $M$. guyanensis, Barbosa et al. (2019) também observaram valores mais baixos desse elemento em outros bivalves coletados na Baía de Todos os Santos, com concentrações, em mg kg-1, de 11,15 em $A$. brasiliana, 30,20 em I. brasiliana, 28,97 em L. pectinata e 11,15 em Trachycardium muricatum (Linnaeus, 1758) (Cardiidae), portanto, todos mais baixos em relação a $C$. rhizophorae. Lino et al. (2016) verificaram $62 \mathrm{mg} \mathrm{kg}^{-1} \mathrm{em} P$. perna da Baía de Guanabara e $52 \mathrm{mg} \mathrm{kg}^{-1} \mathrm{em} N$. nodosus da Ilha Grande e Campolim et al. (2018) observaram 27,11 mg $\mathrm{kg}^{-1}$ em P. perna da Baía de Santos, portanto, todos valores mais baixos aos observados na ostra do presente estudo.

Ainda não está totalmente elucidado o motivo pelo qual ostras acumulam níveis tão altos de zinco. Pedrosa e Cozzolino (2001) consideraram a presença de facilitadores de biodisponibilidade, como a ausência de ácido fítico e a presença de aminoácidos como a glicina, assim como a baixa razão molar ferro: zinco nesses moluscos. Similarmente ao que ocorre com o cobre, o zinco induz a produção da metalotioneínas, assim como participa como componente funcional de várias proteínas, atuando na expressão e regulação gênicas (Miles, Hawksworth, Beattie, \& Rodilla, 2000).

A carência do zinco no organismo humano pode provocar diversos efeitos, como por exemplo, dermatite pustular, alopecia, perda de peso, diarreia, comprometimento imunológico, hipogonadismo e dificuldades na cicatrização de úlceras (Muhamed \& Vadstrup, 2014). A carência deste nutriente é também uma das causas de imunodepressão em crianças (Macêdo et al.,2010).

Os resultados do estudo confirmam que espécies distintas de um mesmo local concentram diferentes níveis de um mesmo elemento metálico. Esse fato pode estar relacionado tanto a fatores metabólicos específicos quanto a características do habitat, sendo que, mais provavelmente, está relacionado a ambos os fatores. No que se refere ao habitat, Cheng, Mana, Nie, \& Wong (2013) afirmaram que o sedimento é a principal fonte de metais para os organismos e que essa matriz os acumula em níveis substancialmente mais altos do que a água. Isso pode explicar, em parte, os níveis expressivos e mais altos do ferro e do manganês em $M$. guyanensis em relação a $C$. rhizophorae, já que a primeira, por ser infaunal, tem um maior contato com o sedimento em relação à segunda espécie, que vive sobre substratos consolidados na faixa entremarés.

As variações nas concentrações de metais entre locais necessitam de investigações adicionais sobre os fatores causais, mas é possível indicar que os níveis mais altos de cobre nas duas espécies e de zinco em C. rhizophorae no L4 (Belmonte), podem ter relação com a atividade de manutenção de pequenas embarcações na faixa litorânea desse município, já que ambos os metais são componentes da maioria das tintas antivegetativas, utilizadas na pintura náutica para evitar o acúmulo de organismos incrustantes (fouling).

\section{Conclusão}

As espécies foram similares em conteúdo de cobre e $M$. guyanensis foi protagonista em relação à concentração de ferro, assim como expressou teores mais altos de manganês em relação a $C$. rhizophorae. Entretanto, $C$. rhizophorae, que teve destaque na concentração de zinco.

\section{Agradecimentos}

À Fundação de Amparo à Pesquisa do Estado da Bahia FAPESB e à Coordenação de Aperfeiçoamento de Pessoal 
de Nível Superior - CAPES, ambas pela concessão da bolsa de doutorado ao primeiro autor e à Comissão Executiva do Plano da Lavoura Cacaueira - CEPLAC, pelo uso do Laboratório de Solos para a digestão das amostras. Aos extrativistas pelo auxílio nas coletas; à Deise Costa e à Julia Benevides, pelo auxílio no processamento laboratorial; ao Vinícius H. C. da Silva, pelo auxílio nos procedimentos e informações analíticas; aos revisores anônimos Ad hoc, pelas contribuições ao manuscrito.

\section{Referências}

Barbosa, I. S., Brito, G. B., Santos, G. L., Santos, L. N., Teixeira, L. S. G., Araujo, R. G. O., \& Korn, M. G. A. (2019). Multivariate data analysis of trace elements in bivalve molluscs: Characterization and food safety evaluation. Food Chemistry, 273(1), 64-70. 10.1016/j.foodchem.2018.02.063

Boehs, G., Luz, M. S. A., \& Andrade, V. R. D. (2019). Molecular identification of cryptic species of oysters (Genus Crassostrea Sacco, 1897) in the Northeast Atlantic coast of Brazil. Boletim do Instituto de Pesca, 45(2), 16. doi: 10.20950/1678-2305.2019.45.2.446

Boehs, G., Villalba, A., Ceuta, L. O., \& Luz, J. R. (2010). Parasites of three Commercially exploited bivalve mollusc species of the estuarine region of the Cachoeira river (Ilhéus, Bahia, Brazil). Journal of Invertebrate Pathology, 103(103), 43-47. doi: 10.1016/j.jip.2009.10.008.

Decreto n. 55.871 de 26 de março de 1965. (1965). Diário Oficial. Brasília.

Cai, C., \& Wang, W. X. (2019). Inter-species difference of copper accumulation in three species of marine mussels: Implication for biomonitoring. Science of the Total Environment, 692(20), 1029-1036. doi: 10.1016/j.scitotenv.2019.07.298

Campolim, M. B., Henriques, M. B., \& Barbieri, E. (2018). Al, Cd, Cr, Cu, Fe, $\mathrm{Mn}, \mathrm{Ni}, \mathrm{Pb}$ and $\mathrm{Zn}$ in mussels collected in Santos Bay, São Paulo, Brazil: Limits required by local legislation. Boletim do Instituto de Pesca, 44(4), 1-8. doi: 10.20950/1678-2305.2018.44.4.374

Cheng, Z., Mana, Y. B., Nie, X. P., \& Wong, M. H. (2013). Trophic relationships and health risk assessments of trace metals in the aquaculture pond ecosystem of Pearl River Delta, China. Chemosphere, 90(7), 21422148. doi: 10.1016/j.chemosphere.2012.11.017

Correia, L. T. A., Veiga, G. R. S., Santos, T. M. M., Cavalcante, C. G., Sawaya, A. L., \& Florêncio, T. M. M. T. (2018). Eficácia do sururu (Mytella falcata) na recuperação de crianças desnutridas, moradoras de favelas de Maceió, Alagoas. Revista Brasileira de Saúde Materno Infantil, 18(01), 223-229. doi: 10.1590/1806-93042018000100011

FAO. [Food and Agriculture Organization] (2020). The state of world fisheries aquaculture 2020. Sustainability in action. Rome. doi: 10.4060/ca9229en.

Gimenez, M. S., Oliveros, L. B. \& Gomez, N. N. (2011). Nutritional deficiencies and phospholipid metabolism. International Journal of Molecular Science, 12(4), 2408-2433. doi: 10.3390/ijms 12042408

Goyer, R. A., \& Clarkson, T. W. (2001). Toxic Effects of Metals. In: Klaasen, C.D., Ed., Casarett and Doullis Toxicology: The Basic Science of Poisons, 6th Edition, McGraw-Hill, New York, 861-867.

LaChance, L. R., \& Ramsey, D. (2018). Antidepressant foods: An evidencebased nutrient profiling system for depression. World Journal of Psychiatry, 8(3), 97-104. doi: 10.5498/wjp.v8.i3.97.

Lindqvist, H. M., Gjertsson, I., Eneljung, T., \& Winkvist, A. (2018). Influence of blue mussel (Mytilus edulis) intake on disease activity in female patients with rheumatoid arthritis: The MIRA randomized cross-over dietary intervention. Nutrients, 10(481), 1-14. doi: 10.3390/nu10040481

Lino, A. S., Galvão, P. M. A., Longo, R. T. L., Azevedo-Silva, C. E., Dorneles, P. R., Torres, J. P. M., \& Malm, O. (2016). Metal bioaccumulation in consumed marine bivalves in Southeast Brazilian coast. Journal of Trace Elements in Medicine and Biology, 34(1), 50-55. doi: 10.1016/j.jtemb.2015.12.004

Macêdo, E. M. C., Amorim, M. A. F., Silva, A. C. S., \& Castro, C. M. M. B. (2010). Efeitos da deficiência de cobre, zinco e magnésio sobre o Sistema imune de crianças com desnutrição grave. Revista Paulista de Pediatria, 28(3), 329-336. doi: 10.1590/s0103-05822010000300012

Maggini, S., Pierre, A., \& Calder, P. C. (2018). Imune function and micronutrient requirements change over the life course. Nutrients, 10 (1531), 1-27. doi: 10.3390/nu10101531.

Miles, A. T., Hawksworth, G. M., Beattie, J. H. \& Rodilla, V. (2000). Induction, regulation, degradation and biological significance of mammalian metallothioneins. Critical Reviews in Biochemistry and Molecular Biology, 35(1), 35-70. doi: 10.1080/10409230091169168
Muhamed, P. K., \& Vadstrup, S. (2014). Zinc is the most important trace element. Ugeskr Laeger, 176(5), 431-433, V11120654.

Pedrosa, L. F. C., \& Cozzolino, S. M. F. (2001). Composição Centesimal e de Minerais de Mariscos Crus e Cozidos da Cidade de Natal/RN. Ciência e Tecnologia de Alimentos, 21(2), 154-157. doi: 10.1590/s0101-20612001000200006

Prato, E., Biandolino, F., Parlapiano, I., Giandomenico, S., Denti, G., Calò, M., Spada, L., \& Di Leo, A. (2019). Proximate, fatty acids and metals in edible marine bivalves from Italian market: Beneficial and risk for consumers health. Science of The Total Environment, 648(1), 153-163. doi: 10.1016/j.scitotenv.2018.07.382

Rodriguéz-Hernandez, A., Zumbado, M., Henríquez-Hernandéz, A., Boada, L. D., \& Luzardo, O. P. (2019). Dietary intake of essential, toxic and potentially toxic elements from mussels (Mytillus spp.) in the Spanish population: A nutritional assessment. Nutrients, 11(864), 1-18. doi: 10.3390/nu11040864

Scheiber, I. F., Mercer, J. F. B., \& Dringen, R. (2014). Metabolism and functions of copper in brain. Progress in Neurobiology, 116(1), 33-57. doi: 10.1016/j.pneurobio.2014.01.002

Tabakaeva, O. V., Tabakaev, A. V., \& Piekoszewski, W. (2018). Nutritional composition and total collagen content of two commercially important edible bivalve molluscs from the Sea of Japan coast. Journal of Food Science and Technology, 55(12), 4877-4886. doi: 10.1007/s13197-018$3422-5$

Weng, N., Jiang, H., \& Wang, W. X. (2019). Novel insights into the role of copper in critical life stages of oysters revealed by High-Resolution Nano SIMS Imaging. Environmental Science \& Technology, 53(24), 14724-14733. doi: 10.1021/acs.est.9b05877

Licença: Creative Commons CC BY NC 4.0 